\title{
Coexistence of sarcoidosis and metastatic lesions: A diagnostic and therapeutic dilemma (Review)
}

\author{
CHRISTOPH SPIEKERMANN ${ }^{1,2}$, MEIKE KUHLENCORD ${ }^{2}$, SEBASTIAN HUSS $^{3}$, \\ CLAUDIA RUDACK $^{1}$ and DANIEL WEISS ${ }^{1}$ \\ ${ }^{1}$ Department of Otorhinolaryngology, Head and Neck Surgery; Institutes of ${ }^{2}$ Immunology \\ and ${ }^{3}$ Pathology, University Hospital Münster, D-48149 Münster, Germany
}

Received May 16, 2017; Accepted September 27, 2017

DOI: $10.3892 / \mathrm{ol} .2017 .7247$

\begin{abstract}
Sarcoidosis, a chronic, inflammatory disease that affects various different organs, is characterized by noncaseating epitheloid granulomas. This systemic inflammatory process is associated with an increased risk of cancer. Several cases of sarcoidosis that mimic metastatic tumor progression in radiological findings have been reported so far. However, there are also cases that have presented a coexistence of sarcoidosis and metastasis, which have caused a diagnostic and therapeutic dilemma. Due to inadequate current therapies, a reliable differentiation between benign and malignant lesions is crucial. This review focuses on the residual risk of the coexistence of metastases within radiological suspicious lesions in patients with a history of solid tumors and sarcoidosis, as well as immunological findings, in order to explain the potential associations. Sarcoidosis has the potential to promote metastasis as it includes tumor-promoting and immune-regulating cell subsets. Notably, myeloid derived suppressor cells may serve a pivotal role in metastatic progression in patients with sarcoidosis. In addition, the present review also evaluates the potential novel diagnostic approaches, which may be able to differentiate between metastatic lesions and sarcoidosis. The risk of coexistent metastasis in sarcoidosis lesions must be considered by clinical practitioners, and a multidisciplinary approach may be required to avoid misdiagnosis and the subsequent unnecessary surgery or insufficient treatments.
\end{abstract}

\section{Contents}

1. Introduction

2. Methods

Correspondence to: Dr Christoph Spiekermann, Institute of Immunology, University Hospital Münster, 21 Röntgenstr, D-48149 Münster, Germany

E-mail: christophOtto.Spiekermann@ukmuenster.de

Key words: review, diagnosis, sarcoidosis, cancer, neoplasm metastasis, tumor microenvironment
3. Results

4. Discussion

5. Conclusion

\section{Introduction}

Sarcoidosis is a chronic, inflammatory, systemic disease affecting primarily the lungs, the mediastinum and the lymphatic system but also salivary glands, heart, nervous system, joints and various other organs (1). Diagnosis depends on the existence of typical clinicoradiological findings in association with noncaseating epitheloid cell granulomas in biopsy and the absence of known, alternative or local causes provoking granulomas (1). Granulomas are nonspecific inflammatory lesions and can occur during mycobacterial, fungal or parasitic infections as well as other diseases like Wegener's granulomatosis (2). Due to the differentiation of granulomas the pathologists play a pivotal role in finding the correct diagnosis (2). In sarcoidosis, granuloma formation is characterized by infiltrating Th1 helper cells and macrophages. The latter show a transformation process into epitheloid cells and can fuse into multinucleate giant cells. Although small amounts of necrosis can be observed, the sarcoid granuloma is referred to the group of nonnecrotizing or noncaseating granulomas (2). Caseating granulomas are typically found in infectious diseases like syphilis or tularemia or infection with tuberculous and nontuberculous mycobacterium (2). In some, especially oncologic patients treated with immunotherapy, noncaseating granulomas can be found although they do not fulfill the criteria for systemic sarcoidosis and are thus referred to as sarcoid-like reactions (3). Due to the toxicity profile of immunotherapies immune-related adverse events can provoke those sarcoid-like reactions which may occur in the organ of tumor origin or in the tumor-draining lymph nodes (3). Sarcoidosis is associated with an increased risk for cancer development in several organs like lung, liver, stomach or for melanoma and lymphoma. Sarcoid-like reactions can be found in $13.8 \%$ of patients with Hodgkin-disease, $7.3 \%$ with non Hodgkin lymphoma and $4.4 \%$ of cases with carcinomas $(4,5)$. Furthermore, simultaneous occurrence of sarcoidosis and cancer is associated with a diminished survival rate (6). Although no increased risk for malignancy in the head and neck has been described so far, 
there are a few cases that report the simultaneous occurrence of sarcoidosis and malignoma of the head and neck (7-9). Notably, in follow-up computed tomography (CT) scans or those done for the detection of primary tumors or metastatic lesions (e.g., 18-Fluorodeoxyglucose positron emission tomography-(PET)-CT, ${ }^{18}$ FDG-PET-CT) sarcoidosis can mimic cancer recurrence or metastatic progress $(10,11)$. However, it is possible that metastatic lesions coexist next to lymph nodes with sarcoid-like lesions and it is unclear whether sarcoidosis has an influence on metastasis of malignoma. Therefore, a review of the current literature was performed to analyze the residual risk of metastasis within radiological suspicious lesions in patients with a history of solid tumors and sarcoidosis.

\section{Methods}

In this review we analyzed reported cases of patients with solid tumors whose staging or follow-up analysis revealed an unclear lymphadenopathy owing to metastasis or sarcoidosis. A systematic literature search was done in Pub Med data base (from inception to April 2017) without any limitation using the terms: Sarcoidosis [title] AND metastasis. All cases with a solid tumor and sarcoidosis were included and the provided information concerning age, gender, tumor region, tumor entity, tumor classification, therapy, as well as information about diagnosis of sarcoidosis were collected. Analyzing the data, the risk of simultaneous occurrence of metastasis and sarcoidosis in concordance to positive radiological and histological findings was elucidated.

Ethical approval. All procedures performed in studies involving human participants were in accordance with the ethical standards of the institutional research committee and with the 1964 Helsinki declaration and its later amendments or comparable ethical standards. Informed consent: Informed consent was obtained from all individual participants included in the study. This article does not contain any studies with animals performed by any of the authors. For this retrospective study and review of anonymized clinical records an ethical permission was not required.

\section{Results}

Review of the literature. Review of the literature revealed 115 cases in Pub Med Data Base based on the search criteria mentioned above. Cases without malignancy were excluded and 59 cases with cancer and sarcoidosis were identified and are listed in Table I. The median age at cancer diagnosis was $49.5+/-13.4$ years and cases of 31 female and 23 male patients were reported. In 5 cases, the sex of the patient was not documented. Systemic sarcoidosis treatment consisted of oral steroids $(n=12)$ or chloroquine $(n=1)$. Local steroids were applied in one case with uveitis. No therapy was initiated in 15 cases and 30 cases did not provide any information about the sarcoidosis therapy approach. However, independent from the treatment strategy the patients' outcome was described to be good with a stable status or complete remission. The most frequent cancer origin was breast $(n=12)$ followed by malignoma of the thyroid gland $(n=8)$. Sarcoidosis occurred in 20 cases after an average of 34.4 months and in 7 cases
10.25 years before diagnosis of malignoma. In most reported cases $(n=24)$ sarcoidosis was revealed simultaneously with diagnosis of malignoma. In 25 cases, surgery was performed to remove the tumor. 24 patients had a combination of surgery and radio-, chemo- or radiochemotherapy. Two cases were only treated by radiochemotherapy and in seven cases, no information about therapeutic aspects concerning the tumor was provided. Occurrence of sarcoidosis after surgery was reported in 3/25 cases and in 17/24 cases after surgery and radio-/chemotherapy. In $17 \%$ of the cases, simultaneous detection of sarcoidosis or sarcoid-like lesions and metastasis was reported $(n=10)$. These reports are now described in more detail.

The first case describes a 50-year-old female patient suffering from ductal invasive breast carcinoma with local lymph node metastasis (pT3pN1). Chest X-ray and ${ }^{18}$ FDG-PET-CT were performed for staging of the tumor and showed a bilateral mediastinal lymphadenopathy and an increased FDG-uptake in supra-diaphragmatic and pelvic lymph nodes. Biopsy of an example lesion obtained sarcoidosis (12). Secondly, an 80-year-old female's CT scan revealed a tumor suspicious mass in the upper lobe of the right lung, multiple smaller nodules and hilar lymphadenopathy. Subsequent biopsy of the mass and a mediastinal lymph node showed just noncaseating granulomas but no malignant cells and led to insufficient treatment. Six months later, symptoms like cough and chest pain were exacerbated and a thoracocentesis revealed adenocarcinoma cells. Further staging examination showed pleural, pericardial and diaphragmatic metastasis. Due to tumor progress, palliative care was initiated (13). The third case describes a 57-year-old female patient who was found to have a choroidal mass in the left eye. Total body gallium 67 scan showed an increased uptake in salivary and lacrimal glands and was misinterpreted as typical for sarcoidosis. Progress of symptoms resulted in enucleation and revealed a choroideal metastasis of a papillary lung carcinoma (14). Sato et al (15) report on a patient with concomitant sarcoidosis and lung adenocarcinoma. Thoracoscopic biopsy of altered lymph nodes did not detect metastasis but sarcoidosis. Surgery was performed and a permanent pathological slide showed that several nodes contained both sarcoidosis and lung cancer metastasis (15). Three other reports describe patients suffering from papillary thyroid carcinoma that underwent thyreoidectomy and modified neck dissection. Pathology revealed concurrent existence of sarcoidosis and regional lymph node metastasis $(10,16,17)$. One patient $(27$-year-old, male) shows a papillary thyroid carcinoma upon a previously diagnosed sarcoidosis. Local lymph nodes contained sarcoidosis mixed with metastasis. A 35-year-old male with a previous history of melanoma developing metastatic involvement and sarcoidosis in regional lymph nodes was described by Chaigne et al (18). Khan and Khan (19) described a 52-year-old patient with cough and chest pain. Radiologic examination showed bilateral hilar lymphadenopathy. Furthermore, within a biopsy of an enlarged lymph node, a metastasis of a left kidney hypernephroma was detected (19).

Taken together, these ten patients had a median age of 49 years ranging from 27 to 80 years at the time point of simultaneous detection of metastasis and sarcoidosis. The gender ratio was $0.6: 1$ (male to female) although no information 


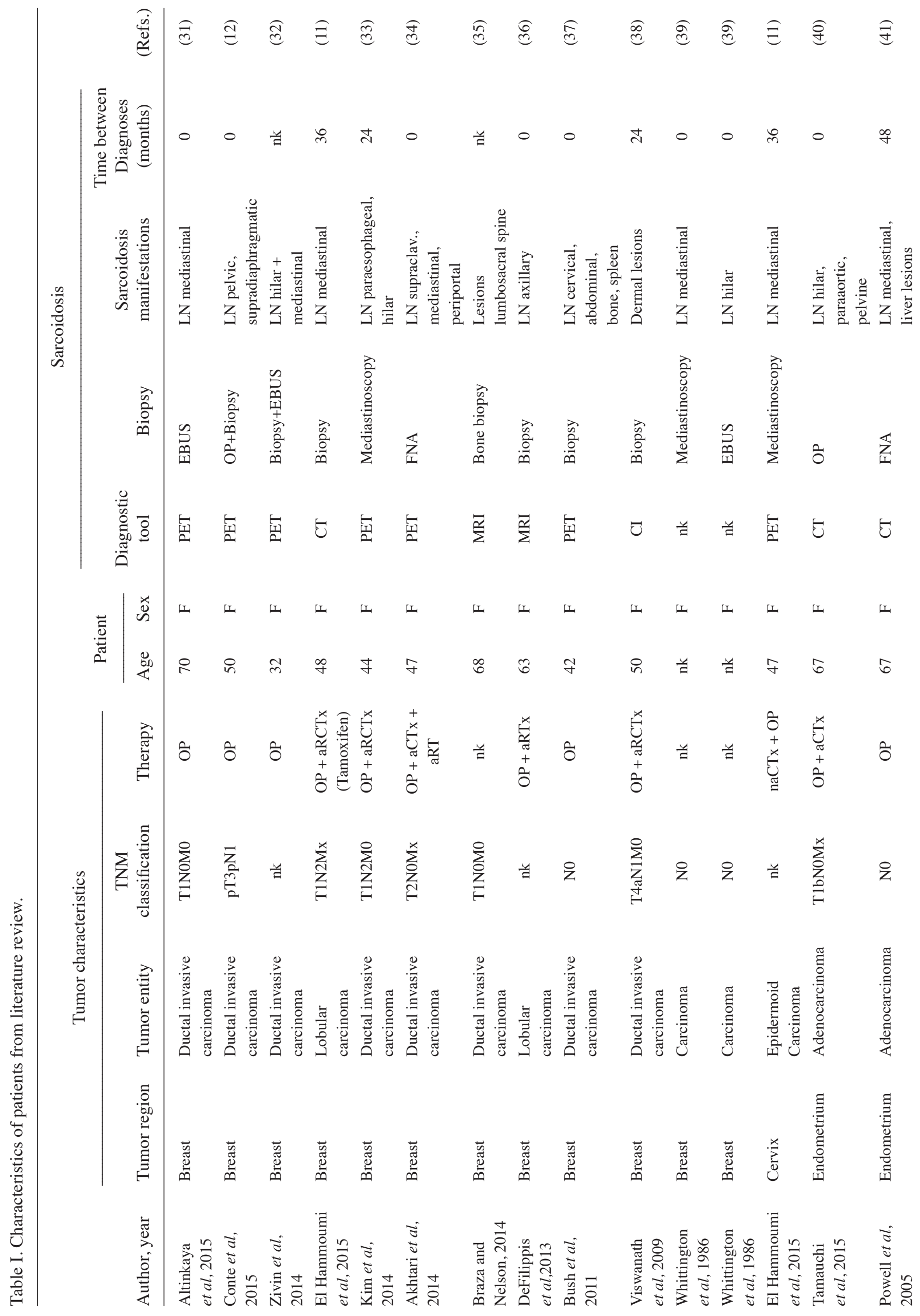




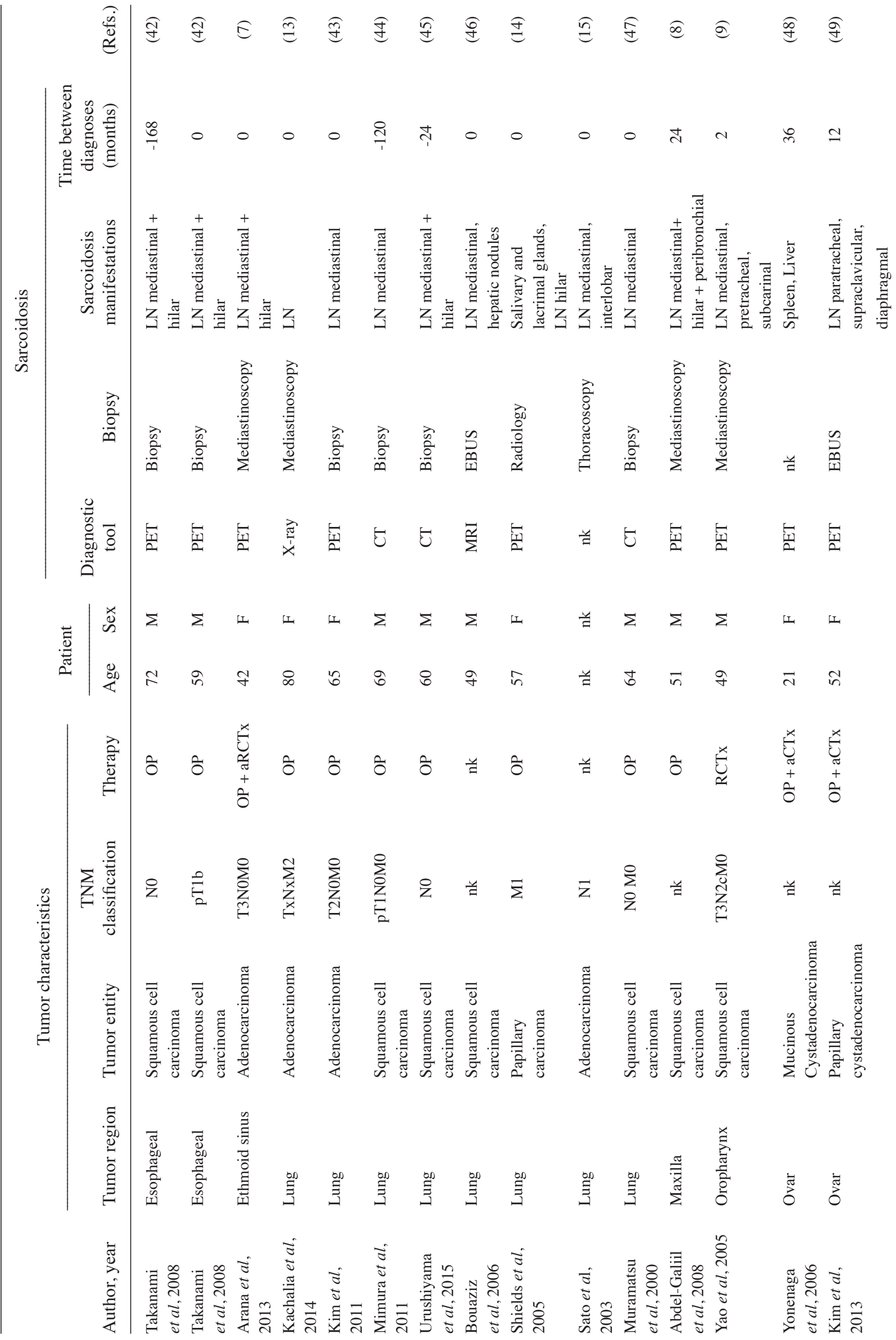




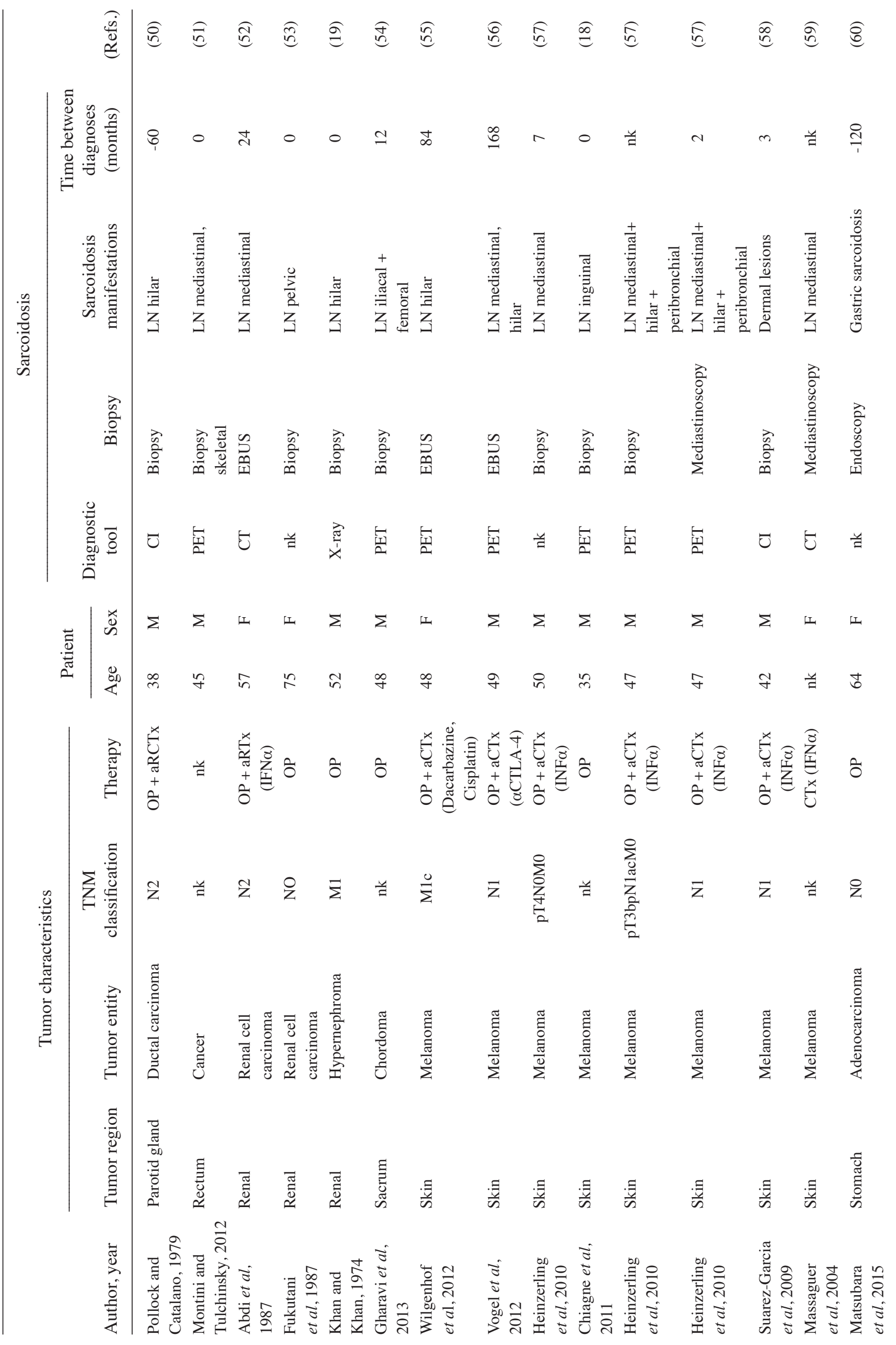




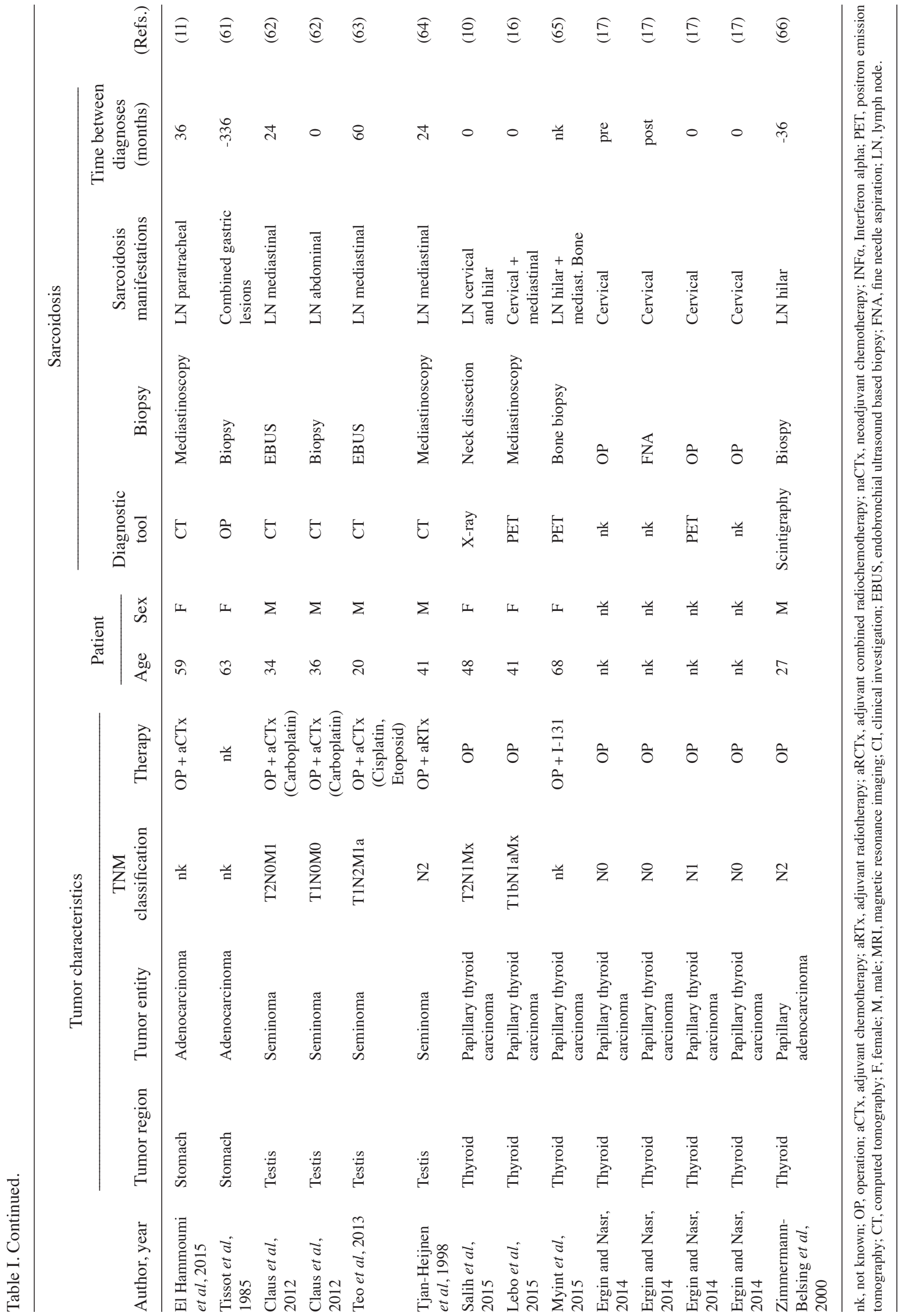


concerning the sex was provided in two cases. In 8 cases (80\%), the metastases were localized in regional lymph nodes whereas just 2 cases showed distant metastases. Furthermore, the region and entity of the associated tumor differed greatly (breast: $n=1$, lung: $n=3$, thyroid: $n=4$, skin: $n=1$, kidney: $n=1$ ).

\section{Discussion}

Sarcoidosis and metastasis. Although there are several published cases concerning coexistence of malignoma and sarcoidosis, the causal relationship between these entities is still unclear. On the one hand, it is possible that patients with sarcoidosis develop malignancies and, on the other hand, there are oncologic patients developing sarcoidosis and sarcoid-like reactions, especially after chemotherapy (3). Several cases describe a mimicking of metastatic disease by sarcoidosis but just a few cases actually report a simultaneous occurrence of sarcoidosis and metastases.

Active sarcoidosis is characterized by an enhanced local expression of T helper 1 (Th1) and T helper 17 (Th17) chemokines and cytokines like IFN- $\gamma$, TNF- $\alpha$, IL-17A and IL-22. In various chronic, autoimmune, inflammatory diseases, such as sarcoidosis, the percentage of IL-17A ${ }^{+} / \mathrm{IFN}-\gamma^{+}$ double-producing Th-cells is increased in peripheral blood and is related to high disease activity. Furthermore, in these pathological conditions, a dysfunctional response of regulatory T-cells (Tregs) has been described that is characterized by an insufficient immunosuppressive function (20). Interestingly, cytotoxic T-lymphocyte antigen 4 (CTLA-4) expression is decreased while PD-1 (programmed death-1) expression is increased in Th17-cells in the mediastinal lymph nodes during sarcoidosis (20). CTLA-4 is present on Th-cells and mediates an inhibitory effect on further $\mathrm{T}$ cells responses. Hence, a diminished CTLA-4 level maintains inflammatory reactions. Similarly, PD-1 is expressed on the surface of T-cells upon activation and is involved in limiting inflammatory reactions (21). Its ligand, PD-1L, can be found on tumor cells and provokes upregulation of PD-1 in T cells. Consequently, activation of tumor antigen-specific T-cells in pancreatic adenocarcinoma is inhibited (22). Hence, the bivalent adaptive immune response in patients with sarcoidosis and metastasis promotes both pathological conditions by maintaining sarcoidosis-related inflammation due to the decreased anti-inflammatory CTLA-4 expression while limiting tumor-specific $\mathrm{T}$ cell activation, marked by an increased PD-1 expression, that enables tumor escape from the immune system and metastasis. Increased PD-1 expression on T-cells in sarcoidosis lymph nodes could thus be a possible predictor of metastasis on the basis of sarcoidosis. Furthermore, myeloid-derived suppressor cells (MDSC) might play a pivotal role in the pathogenic association between sarcoidosis and metastasis. MDSC pursue immunoregulatory and T-cell suppressive functions (23). Although it has not been described yet, an influence of MDSC on sarcoidosis is assumable because of their important role in other inflammatory diseases (24). Th-17 cells are the main source of IFN- $\gamma$ production in sarcoidosis and IFN- $\gamma$ induces MDSC differentiation and promotes their immunosuppressive function $(20,25)$. In cancer models, MDSC accumulation was promoted by several cytokines and growth factors, such as IL-6, IL-1ß and S100A8/A9, resulting in an anti-inflammatory tumor microenvironment (23). MDSC themselves express cytokines and chemokines like IL-6, TNF, IL-1 $\beta$, IL-23 and S100A9 and have the potential to attract both further myeloid cells and tumor cells (26). Furthermore, in a melanoma and lung carcinoma mouse model, S100A9 expressing MDSC were identified as important players in enabling tumor metastasis (27). Consequently, they are generally recognized as dominant tumor-promoting forces (28). Patients suffering from sarcoidosis have increased serum levels of S100A8/A9 and an enhanced cytoplasmatic S100A8/A9 expression in monocytes and multinuclear giant cells in granulomas (29). Because of the sarcoidosis-related inflammation, marked by the expression of IFN- $\gamma$, IL-6, IL-23 and S100A8/A9, we can suspect a similar microenvironment to tumors that are characterized by an increased accumulation and immunosuppressive function of MDSC. Accordingly, we can assume that sarcoidosis has the potential to promote metastasis by inducing tumor-promoting and immune-regulating cell subsets. Further analysis is necessary to verify the influence of MDSC on sarcoidosis as well as the cellular immune response concerning the association between sarcoidosis and metastasis.

Differentiation between benign and malignant lesions. PET-CT scan is a very useful diagnostic tool to identify malignant lesions with a sensitivity between 47 and $100 \%$ and a specificity of 86-100\% (30). Unfortunately, elevated FDG uptake can also be detected in inflammatory diseases such as sarcoidosis causing a diagnostic dilemma. The case of a 61-year-old carpenter with a history of adenocarcinoma of the paranasal sinus and simultaneous occurrence of multiple cervical metastases and sarcoidosis detected during follow-up investigation impressively demonstrates the risk to overlook metastatic lesions within sarcoidosis (Fig. 1). Especially PET scans for staging or restaging of oncologic diseases supply important information about tumor progression. Decisions on curative or palliative therapy are based on this information, emphasizing the importance to avoid misdiagnosis. Inclusion of an additional tracer would be helpful to differentiate between inflammation and tumor. F18-labeled 3'deoxy-3'fluorothymidine (FLT) is such a promising tracer to minimize diagnostic and subsequent therapeutic mistakes. By measuring DNA synthesis instead of metabolic activity that seems to be more specific to detect tumor diseases, FLT might be a useful candidate to discriminate between tumor and sarcoidosis lesions (7).

An additional helpful tool to discriminate inflammatory bone marrow involvement, like skeletal sarcoidosis, from metastatic disease might be the diffusion whole-body magnetic resonance imaging (b-values $50-900 \mathrm{~s} / \mathrm{mm}^{2}$ ). In contrast to malignant lesions (cut-off value of $774 \mu \mathrm{m}^{2} / \mathrm{s}$ ), sarcoidosis or other inflammatory skeletal reactions show high signal intensity on diffusion-weighted images and a lower apparent diffuse coefficient (ADC) (12).

Bioptical evaluation of radiologically altered lymph nodes is necessary for selection of appropriate oncological treatment strategy. However, examination of each PET-CT positive lesion is not feasible and the chance to detect metastatic lesions next to sarcoidosis is rather rare (Fig. 2). Thus, even if pathological findings were suspicious of sarcoidosis, concomitant metastasis cannot be certainly excluded. Hence, correlation between 

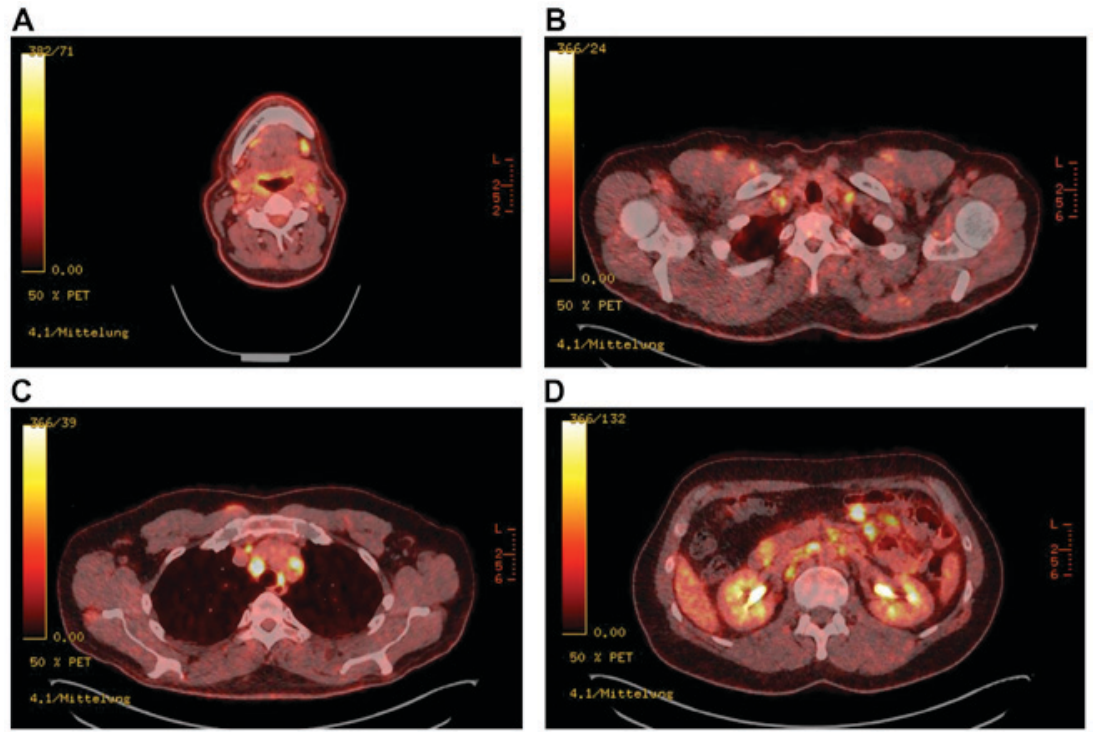

Figure 1. PET-CT showing multiple high uptake lesions: 18-Fluorodeoxyglucose positron emission tomography and computed tomography ( ${ }^{18}$ FDG-PET-CT) of a patient with a history of paranasal sinus adenocarcinoma shows increased uptake of (A) submandibular, (B) cervical, (C) mediastinal and (D) mesenterial lymph nodes suspicious of multiple metastatic lesions. Bioptical examination revealed cervical metastases of the known adenocarcinoma and mediastinal sarcoid-like lesions without malignancy. Courtesy of Professor W. Heindel, Institute of Clinical Radiology, University Hospital Münster. ${ }^{18}$ FDG-PET-CT, 18 -fluorodeoxyglucose-positron emission tomography-computed tomography.
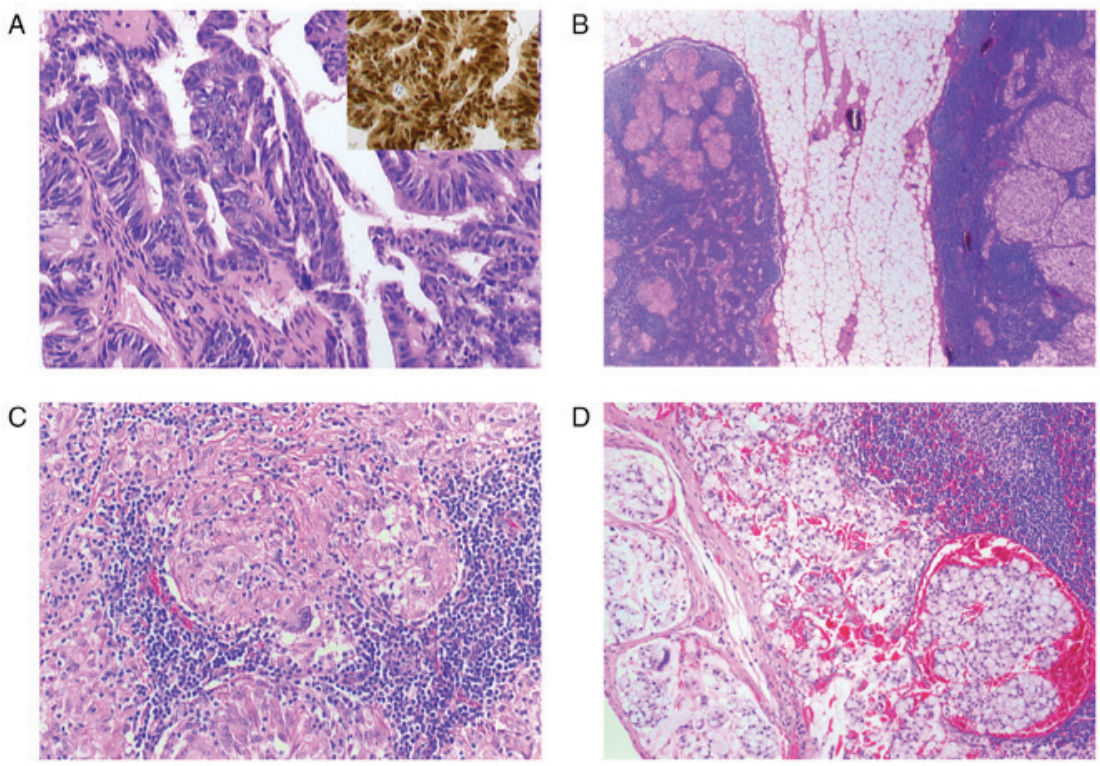

Figure 2. (A-D) Histological findings of a resected paranasal sinus adenocarcinoma and lymph node. (A) Microphotograph shows the adenocarcinoma with atypical tumor cells (H\&E; magnification, x400) and CDX2 nuclear positive cells, characteristic for intestinal-type adenocarcinoma (inset, H\&E, magnification, $\mathrm{x} 400$ ). (B) Two lymph nodes surrounded by perinodal fat tissue. The right node with noncaseating epitheloid granulomas and the left one with metastatic lesions (H\&E, magnification, x5). (C) Lymph node with noncaseating epitheloid granuloma in higher magnification (H\&E, magnification, x200). Multinuclear giant cells are identifiable. (D) Metastatic carcinoma infiltration in a lymph node (H\&E, magnification, x200). H\&E, hematoxylin and eosin; CDX2, caudal type homeobox 2 .

the radiological and histological findings with the probability of distant or local metastasis corresponding to the tumor entity is important for the careful assessment of the residual metastasis risk.

\section{Conclusion}

Correlation between PET-Scan, histological findings and knowledge about typical tumor behavior is necessary to avoid misdiagnosis. Nevertheless, a residual risk of overlooking metastases in systemic inflammatory diseases still remains existent. Therefore, it is important for clinical practice to be aware of the simultaneous occurrence of sarcoidosis and metastatic malignancy. Further cell subset analysis in these pathologies might additionally reveal immunological distinct cell populations as useful markers to distinguish between sarcoidosis, cancer and the coexistence of these two and help in overcoming the current diagnostic dilemma. 


\section{Acknowledgements}

This study was supported by a fellowship from the medical faculty of the University of Münster, Germany to C. Spiekermann.

\section{References}

1. Statement on sarcoidosis. Joint Statement of the American Thoracic Society (ATS), the European Respiratory Society (ERS) and the World Association of Sarcoidosis and Other Granulomatous Disorders (WASOG) adopted by the ATS Board of Directors and by the ERS Executive Committee, February 1999. Am J Respir Crit Care Med 160: 736-755, 1999.

2. Rosen Y: Pathology of sarcoidosis. Semin Respir Crit Care Med 28: 36-52, 2007.

3. Cohen PR and Kurzrock R: Sarcoidosis and malignancy. Clin Dermatol 25: 326-333, 2007.

4. Brincker H: Sarcoid reactions in malignant tumours. Cancer Treat Rev 13: 147-156, 1986.

5. Askling J, Grunewald J, Eklund A, Hillerdal G and Ekbom A: Increased risk for cancer following sarcoidosis. Am J Respir Crit Care Med 160: 1668-1672, 1999.

6. Boffetta P, Rabkin CS and Gridley G: A cohort study of cancer among sarcoidosis patients. Int J Cancer 124: 2697-2700, 2009.

7. Arana Yi C, McCue P, Rosen M, Machtay M, Axelrod R and Morris GJ: Sarcoidosis mimicking metastatic bone disease in head and neck cancer. Semin Oncol 40: 529-534, 2013.

8. Abdel-Galiil K, Anand R, Sharma S, Brennan PA, Ramchandani PL and Ilankovan V: Incidence of sarcoidosis in head and neck cancer. Br J Oral Maxillofac Surg 46: 59-60, 2008

9. Yao M, Funk GF, Goldstein DP, DeYoung BR and Graham MM: Benign lesions in cancer patients: Case 1. Sarcoidosis after chemoradiation for head and neck cancer. J Clin Oncol 23: 640-641, 2005

10. Salih AM, Fatih SM and Kakamad FH: Sarcoidosis mimicking metastatic papillary thyroid cancer. Int J Surg Case Rep 16: 71-72, 2015.

11. El Hammoumi M, El Marjany M, Moussaoui D, Doudouh A, Mansouri $\mathrm{H}$ and Kabiri el $\mathrm{H}$ : Mediastinal sarcoidosis mimicking lymph malignancy recurrence after anti-neoplastic therapy. Arch Bronconeumol 51: e33-e35, 2015 (In English, Spanish).

12. Conte G, Zugni F, Colleoni M, Renne G, Bellomi M and Petralia G: Sarcoidosis with bone involvement mimicking metastatic disease at (18)F-FDG PET/CT: Problem solving by diffusion whole-body MRI. Ecancermedicalscience 9: 537, 2015

13. Kachalia AG, Ochieng P, Kachalia K and Rahman H: Rare coexistence of sarcoidosis and lung adenocarcinoma. Respir Med Case Rep 12: 4-6, 2014.

14. Shields JA, Shields CL and Eagle RC Jr: Choroidal metastasis from lung cancer masquerading as sarcoidosis. Retina 25: 367-370, 2005.

15. Sato Y, Sasano S, Oyama K, Sakuraba M, Onuki T and Nitta S: Lung cancer associated with sarcoidosis. Jpn J Thorac Cardiovasc Surg 51: 21-24, 2003.

16. Lebo NL, Raymond F and Odell MJ: Selectively false-positive radionuclide scan in a patient with sarcoidosis and papillary thyroid cancer: A case report and review of the literature. J Otolaryngol Head Neck Surg 44: 18, 2015.

17. Ergin AB and Nasr CE: Thyroid cancer \& sarcoidosis. Sarcoidosis Vasc Diffuse Lung Dis 31: 239-243, 2014.

18. Chaigne B, Perrinaud A, Penaud A, Machet MC, Venel Y, Marchand-Adam S and Machet L: Melanoma lymph node metastasis occurring simultaneously with multifocal sarcoidosis affecting lymph nodes and the lung: A diagnostic pitfall. Eur J Dermatol 21: 798-799, 2011.

19. Khan A and Khan FA: Hypernephroma: A rare cause of bilateral adenopathy, and an example of the importance of tissue diagnosis in suspected cases of sarcoidosis. Chest 66: 722-723, 1974.

20. Broos CE, Hendriks RW and Kool M: T-cell immunology in sarcoidosis: Disruption of a delicate balance between helper and regulatory T-cells. Curr Opin Pulm Med 22: 476-483, 2016.

21. Hashemi-Sadraei N, Sikora AG and Brizel DM: Immunotherapy and checkpoint inhibitors in recurrent and metastatic head and neck cancer. Am Soc Clin Oncol Educ Book 35: e277-e282, 2016.

22. Seo YD and Pillarisetty VG: T-cell programming in pancreatic adenocarcinoma: A review. Cancer Gene Ther 24: 106-113, 2017.

23. Crook KR and Liu P: Role of myeloid-derived suppressor cells in autoimmune disease. World J Immunol 4: 26-33, 2014.
24. Nagaraj S, Collazo M, Corzo CA, Youn JI, Ortiz M, Quiceno D and Gabrilovich DI: Regulatory myeloid suppressor cells in health and disease. Cancer Res 69: 7503-7506, 2009.

25. Zhao Y, Wu T, Shao S, Shi B and Zhao Y: Phenotype, development, and biological function of myeloid-derived suppressor cells. Oncoimmunology 5: e1004983, 2015.

26. Liu Y, Kosaka A, Ikeura M, Kohanbash G, Fellows-Mayle W, Snyder LA and Okada H: Premetastatic soil and prevention of breast cancer brain metastasis. Neuro Oncol 15: 891-903, 2013.

27. Yang Q, Li X, Chen H, Cao Y, Xiao Q, He Y, Wei J and Zhou J: IRF7 regulates the development of granulocytic myeloid-derived suppressor cells through S100A9 transrepression in cancer. Oncogene 36: 2969-2980, 2017.

28. Zamarron BF and Chen W: Dual roles of immune cells and their factors in cancer development and progression. Int J Biol Sci 7: 651-658, 2011.

29. Terasaki F, Fujita M, Shimomura H, Tsukada B, Otsuka K, Otsuka K, Katashima T, Ikemoto M and Kitaura Y: Enhanced expression of myeloid-related protein complex (MRP8/14) in macrophages and multinucleated giant cells in granulomas of patients with active cardiac sarcoidosis. Circ J 71: 1545-1550, 2007.

30. Zanation AM, Sutton DK, Couch ME, Weissler MC, Shockley WW and Shores CG: Use, accuracy, and implications for patient management of [18F]-2-fluorodeoxyglucose-positron emission/computerized tomography for head and neck tumors. Laryngoscope 115: 1186-1190, 2005.

31. Altinkaya M, Altinkaya N and Hazar B: Sarcoidosis mimicking metastatic breast cancer in a patient with early-stage breast cancer. Ulus Cerrahi Derg 32: 71-74, 2015.

32. Zivin S, David O and Lu Y: Sarcoidosis mimicking metastatic breast cancer on FDG PET/CT. Intern Med 53: 2555-2556, 2014

33. Kim HS, Lee SY, Oh SC, Choi CW, Kim JS and Seo JH: Case report of pulmonary sarcoidosis suspected to be pulmonary metastasis in a patient with breast cancer. Cancer Res Treat 46: 317-321, 2014.

34. Akhtari M, Quesada JR, Schwartz MR, Chiang SB and Teh BS: Sarcoidosis presenting as metastatic lymphadenopathy in breast cancer. Clin Breast Cancer 14: e107-e110, 2014.

35. Braza DW and Nelson PA: Vertebral sarcoidosis masquerading as breast metastasis. Am J Phys Med Rehabil 93: 274, 2014.

36. DeFilippis EM and Arleo EK: New diagnosis of sarcoidosis during treatment for breast cancer, with radiologic-pathologic correlation. Clin Imaging 37: 762-766, 2013.

37. Bush E, Lamonica D and O'Connor T: Sarcoidosis mimicking metastatic breast cancer. Breast J 17: 533-535, 2011.

38. Viswanath L, Pallade S, Krishnamurthy B, Naveen T, Preethi BL, Pramod KP, Reddy O and Padma G: Darier-roussy sarcoidosis mimicking metastatic breast cancer. Case Rep Oncol 2: 251-254, 2009.

39. Whittington R, Lazarus A, Nerenstone S and Martin A: Sarcoidosis developing during therapy for breast cancer. Chest 89: 762-763, 1986.

40. Tamauchi S, Shimomura Y and Hayakawa H: Endometrial cancer with sarcoidosis in regional lymph nodes: A case report. Case Rep Oncol 8: 409-415, 2015.

41. Powell JL, Cunill ES, Gajewski WH and Novotny DB: Sarcoidosis mimicking recurrent endometrial cancer. Gynecol Oncol 99: 770-773, 2005.

42. Takanami K, Kaneta T, Yamada T, Kinomura S, Yamada S, Fukuda $\mathrm{H}$ and Takahashi S: FDG PET for esophageal cancer complicated by sarcoidosis mimicking mediastinal and hilar lymph node metastases: Two case reports. Clin Nucl Med 33: 258-261, 2008.

43. Kim JJ, Park JK, Wang YP, Choi SH and Jo KH: Lung cancer associated with sarcoidosis - A case report. Korean J Thorac Cardiovasc Surg 44: 301-303, 2011.

44. Mimura K, Mochizuki Y, Nakahara Y, Kawamura T, Sasaki S and Katsuda R: A case of primary lung cancer with swollen mediastinal lymph nodes due to pre-existing sarcoidosis. Nihon Kokyuki Gakkai Zasshi 49: 208-213, 2011 (In Japanese).

45. Urushiyama H, Yamauchi Y, Suzuki S, Sunohara M, Kouyama T, Ohishi N, Fukami T, Nakajima J, Ushiku T, Oota S, et al: Case of sarcoidosis with squamous cell carcinoma which originated from solitary bronchial papilloma. Nihon Kokyuki Gakkai Zasshi 48: 815-820, 2010 (In Japanese).

46. Bouaziz H, Kaffel N, Charfi N, Fourati M, Abid H and Abid M: Panhypopituitarism revealing metastasis of small-cell lung carcinoma associated with sarcoidosis. Ann Endocrinol (Paris) 67: 259-264, 2006 (In French). 
47. Muramatsu M, Kuriyama M, Takahashi K, Miyamoto $\mathrm{H}$, Uekusa T, Danbara T and Fukuchi Y: A case of resected squamous cell carcinoma of the lung complicated with sarcoidosis. Nihon Kokyuki Gakkai Zasshi 38: 720-725, 2000 (In Japanese).

48. Yonenaga Y, Kushihata F, Inoue H, Watanabe J, Tohyama T, Sugita A and Takada Y: Sarcoidosis manifesting as hepatic and splenic nodules mimicking ovarian cancer metastases: A case report. Oncol Lett 10: 2166-2170, 2015.

49. Kim MH, Lee K, Kim KU, Park HK, Lee MK and Suh DS: Sarcoidosis mimicking cancer metastasis following chemotherapy for ovarian cancer. Cancer Res Treat 45: 354-358, 2013.

50. Pollock JL and Catalano E: Metastatic ductal carcinoma of the parotid gland in a patient with sarcoidosis. Arch Dermatol 115 1098-1099, 1979.

51. Montini KM and Tulchinsky M: False-positive bone metastases on PET/CT secondary to sarcoidosis in a patient with rectal cancer. Clin Nucl Med 37: 307-310, 2012.

52. Abdi EA, Nguyen GK, Ludwig RN and Dickout WJ: Pulmonary sarcoidosis following interferon therapy for advanced renal cell carcinoma. Cancer 59: 896-900, 1987.

53. Fukutani K, Kawabe K, Moriyama N, Kitamura $\mathrm{T}$ and Murakami T: Carcinoma of the renal pelvis and bladder associated with sarcoidosis: A case report. Urol Int 42: 224-226, 1987.

54. Gharavi $\mathrm{MH}, \mathrm{Wu} \mathrm{HH}$ and Toms SA: High fluorodeoxyglucose ((18)F)PET-uptake lymph nodes in a patient with chordoma: Tumor metastasis or sarcoidosis? Am J Case Rep 14: 373-375, 2013.

55. Wilgenhof S, Morlion V, Seghers AC, Du Four S, Vanderlinden E, Hanon S, Vandenbroucke F, Everaert H and Neyns B: Sarcoidosis in a patient with metastatic melanoma sequentially treated with anti-CTLA-4 monoclonal antibody and selective BRAF inhibitor. Anticancer Res 32: 1355-1359, 2012.

56. Vogel WV, Guislain A, Kvistborg P, Schumacher TN, Haanen JB and Blank CU: Ipilimumab-induced sarcoidosis in a patient with metastatic melanoma undergoing complete remission. J Clin Oncol 30: e7-e10, 2012.
57. Heinzerling LM, Anliker MD, Muller J, Schlaeppi M and von Moos R: Sarcoidosis induced by interferon- $\alpha$ in melanoma patients: Incidence, clinical manifestations, and management strategies. J Immunother 33: 834-839, 2010.

58. Suarez-Garcia C, Pérez-Gil A, Pereira-Gallardo S, Codes-Villena M, García-Escudero A and Miguel Camacho F: Interferon-induced cutaneous sarcoidosis in melanoma. Melanoma Res 19: 391-394, 2009.

59. Massaguer S, Sánchez M and Castel T: Mediastinal sarcoidosis induced by high-dose alpha-2-interferon therapy in a patient with malignant melanoma. Eur Radiol 14: 1716-1717, 2004

60. Matsubara T, Hirahara N, Hyakudomi R, Fujii Y, Kaji S, Taniura T and Tajima Y: Early gastric cancer associated with gastric sarcoidosis. Int Surg 100: 949-953, 2015.

61. Tissot E, Bringeon G, Berger F and Kalb JC: Cancer and gastric sarcoidosis. J Chir (Paris) 122: 479-481, 1985 (In French).

62. Claus F, De Wever L and Moerman P: Coincidence of seminoma and sarcoidosis in two patients presenting with peritoneal surface disease. Int J Urol 19: 1126, 2012.

63. Teo M, McCarthy JE, Brady AP, Curran DR and Power DG: A case of sarcoidosis in a patient with testicular cancer post stem cell transplant. Acta Oncol 52: 869-871, 2013.

64. Tjan-Heijnen VC, Vlasveld LT, Pernet FP, Pauwels P and De Mulder PH: Coincidence of seminoma and sarcoidosis: A myth or fact? Ann Oncol 9: 321-325, 1998.

65. Myint ZW and Chow RD: Sarcoidosis mimicking metastatic thyroid cancer following radioactive iodine therapy. J Community Hosp Intern Med Perspect 5: 26360, 2015.

66. Zimmermann-Belsing $\mathrm{T}$, Christensen L, Hansen HS, Kirkegaard J, Blichert-Toft $M$ and Feldt-Rasmussen U: A case of sarcoidosis and sarcoid granuloma, papillary carcinoma, and Graves' disease in the thyroid gland. Thyroid 10: 275-278, 2000. 